\title{
Effect of Foot Orthoses in Children With Symptomatic Flexible Flatfoot Based on Ultrasonography of the Ankle Invertor and Evertor Muscles
}

\author{
Dong Joon Cho, MD, So Young Ahn, MD, PhD, Soo-Kyung Bok, MD, PhD
}

Department of Rehabilitation Medicine, College of Medicine, Chungnam National University Hospital, Daejeon, Korea

\begin{abstract}
Objective To examine the changes in the cross-sectional area (CSA) ratio of the ankle invertors and evertors following rigid foot orthosis (RFO) application in children with symptomatic flexible flatfoot and to determine the correlation between the degree of change in CSA ratio and pain-severity after RFO application.

Methods We included 24 children with symptomatic flexible flatfoot without comorbidities and measured the CSAs of tibialis anterior (TA), tibialis posterior (TP), and peroneus longus (PL) using ultrasonography, resting calcaneal stance position (RCSP) angle, calcaneal pitch (CP), Meary's angle, talonavicular coverage angle, and talocalcaneal angle using radiography, and foot function index (FFI) at baseline and 12 months after RFO application. We analyzed 48 data by measuring both feet of 24 children. The CSA ratios, the ratio of CSA of each muscle to the sum of CSA of TA, TP, and PL, were also compared. Correlations between the degree of change in FFI, each muscle's CSA ratio, RCSP angle, and radiographic measurements were investigated.

Results Following RFO application, significant increase in the PL ratio and CP and significant decrease in the RCSP angle, FFI total, pain, and disability scores were observed. The degree of change in the total score, pain, and disability score of FFI were significantly correlated with the degree of change in the PL ratio and RCSP angle.

Conclusion RFOs applied to children with symptomatic flexible flatfoot might reduce the compensatory activities of the ankle invertors, thereby increasing the PL ratio, and pain decreases as the PL ratio increases.
\end{abstract}

Keywords Foot orthoses, Ankle, Ultrasonography, Flatfoot, Child

Received July 23, 2021; Revised September 17, 2021; Accepted September 27, 2021; Published online December 31, 2021 Corresponding author: Soo-Kyung Bok

Department of Rehabilitation Medicine, College of Medicine, Chungnam National University Hospital, 282 Munhwa-ro, Jung-gu, Daejeon 35015, Korea. Tel: +82-42-338-2460, Fax: +82-42-338-2461, E-mail: skbok111@gmail.com

ORCID: Dong Joon Cho (https://orcid.org/0000-0002-2355-1580); So Young Ahn (https://orcid.org/0000-0002-7447-0617); Soo-Kyung Bok (https:// orcid.org/0000-0002-8957-2827).

() This is an open-access article distributed under the terms of the Creative Commons Attribution Non-Commercial License (http://creativecommons.org/ licenses/by-nc/4.0) which permits unrestricted noncommercial use, distribution, and reproduction in any medium, provided the original work is properly cited. Copyright $\odot 2021$ by Korean Academy of Rehabilitation Medicine 


\section{INTRODUCTION}

Flatfoot or pes planus refers to the loss of the medial longitudinal arch (MLA) [1]. The MLA is composed of the calcaneus, talus, navicular bone, and first metatarsal bone of the foot and reduces the impact on the foot while walking [2]. There are two types of flatfoot: flexible and rigid. In flexible flatfoot, the MLA collapses in the weightbearing foot during a loading response but reappears when the foot is no longer bearing weight [1]. The flatfoot prevalence is $44 \%$ among children aged 3-6 years, $13.4 \%$ $27.6 \%$ among primary-school aged children, and $13.6 \%$ in adolescents aged 18-21 years; flatfoot is more prevalent in younger age groups and become less prevalent with age $[1,3,4]$. Since a study reported that flatfoot can cause complications, including foot, knee, and back pain, and scoliosis [5-7], the interest in the diagnosis and treatment of flatfoot has been growing, specifically among parents.

Several studies have reported differences in foot kinematics during gait between people with flatfoot and those without it [8-11]. Studies examining the kinematics of patients with flatfoot using surface electromyography have reported that peroneus longus (PL) activity is reduced during gait [9] and that the thickness and cross-sectional area (CSA) of the PL are reduced in patients with flatfoot $[12,13]$. These results suggest that flattening of the MLAs and abduction of the forefeet in patients with flexible flatfoot is associated with PL underactivity. Using ultrasound, Sakamoto and Kudo [2] found that the activity of the abductor hallucis (AH), which is a forefoot adductor, increases to compensate for MLA collapse, and its CSA increases in patients with flatfoot. Shin et al. [14] measured the CSAs of the tibialis anterior (TA) and tibialis posterior (TP) (ankle invertors) and PL (ankle evertor) in flatfoot patients using ultrasonography and compared the CSA ratio, which is the ratio of each muscle's CSA to the total CSA of the TA, TP, and PL, combined. The CSA ratios of the TA and TP were higher and those of the PL were lower in individuals with flexible flatfoot than in those without. Thus, people with flexible flatfoot maintain the alignment of their feet and legs when walking using an ankle invertor muscle to compensate for MLA collapse during weight-bearing, which may in turn cause hypertrophy of ankle inverters, such as the TA and TP.

Flatfoot has been reported to induce pain and fatigue during walking. However, the direct cause or mechanism by which flatfoot causes pain is unclear [14-16]. Most patients with flatfoot report pain in the sole and calves, and some report pain in the front and back of the knee and ankle $[17,18]$. While a few studies have reported no correlations between the arch of the foot and pain among patients with flexible flatfoot [19], a study has shown significant correlations between pain severity and the PL ratio; this PL ratio is the ratio of CSA of the PL to the total CSA of the TA, TP, and PL, combined [14]. Conservative treatments are often administered to reduce pain and improve gait disability in patients with symptomatic flatfoot, including stretching exercises and foot orthoses [15,2022]. However, whether a foot orthosis can effectively treat flatfoot remains to be clearly established $[23,24]$. Nonetheless, a recent study by Youn et al. [25] showed that rigid foot orthoses (RFOs) improve foot posture and radiographic indices in patients with flexible flatfoot.

Based on the aforementioned findings, we hypothesized that RFOs would prevent the overpronation of the foot during the loading response, thereby reducing hypertrophy of the ankle invertors and changing the CSA ratios of the TA, TP, and PL. Thus, this study aimed to investigate changes in the CSA ratios of the ankle invertors and evertors, which are ankle-stabilizing muscles, following RFO application in patients with symptomatic flatfoot and to examine the correlations between the degree of change in these parameters.

\section{MATERIALS AND METHODS}

\section{Participants}

This longitudinal study was conducted between July 2019 and March 2021. We recruited children between the ages of 10 and 18 years who visited the outpatient Department of Rehabilitation Medicine at Chungnam National University Hospital and were diagnosed with symptomatic flexible flatfoot. The diagnostic criteria for symptomatic flatfoot were as follows: (1) resting calcaneal stance position (RCSP) angle <- $4^{\circ}[10]$; (2) calcaneal pitch (CP) $<18^{\circ}$ and Meary's angle (MA) $>4^{\circ}$ on foot radiographs; and (3) numeric rating scale score for foot or leg pain $\geq 2$. Patients with gait disabilities or foot deformities, including bony abnormalities such as tarsal coalition, due to neurological or orthopedic conditions were excluded. Genetic diseases such as Ehlers-Danlos syndrome, benign joint hypermobility syndrome, and Marfan syn- 
drome that can cause ligament laxity were also excluded. This study received approval from the Institutional Review Board of Chungnam National University Hospital (No. 2020-02-047). Because the participants were minors, we explained the overall content of the study to their nearest of kin (participants' parents) who provided consent on the minors' behalf to participate in the study.

\section{Study design}

The RCSP angle, radiographic and ultrasonographic measurements, and foot function index (FFI) were assessed during the first outpatient visit. Patients were prescribed RFOs after undergoing initial evaluation. After 12 months of applying the RFO, re-evaluation of the same metrics was conducted.

\section{Outcome measurements}

The primary outcome of this study was the changes in the CSA ratios of the ankle invertors and evertors, which were measured using ultrasonography. The secondary outcomes were the changes in the RCSP angle, changes in radiographic measurements including $\mathrm{CP}, \mathrm{MA}$, talonavicular coverage angle (TNCA), and talocalcaneal angle (TCA); and FFI, which assesses symptoms such as pain and gait disability.

\section{Primary outcome}

Ultrasonographic measurements: A physiatrist with at least 2 years of clinical experience in ultrasound examination measured the CSAs of the TA, TP, and PL using the ACUSON S2000 ultrasound unit (Siemens, Mountain View, CA, USA), with a linear probe with a frequency of 9-14 MHz. The CSA of each muscle was measured using a method described in previous studies [12,14]. The measurement locations were as follows: TA, one-third from the tibial tuberosity between the tibial tuberosity and the medial malleolus; TP, at the middle between the medial malleolus and the tibial tuberosity; and PL, one-third from the tibial tuberosity between the tibial tuberosity and the lateral malleolus (Fig. 1). There are two methods
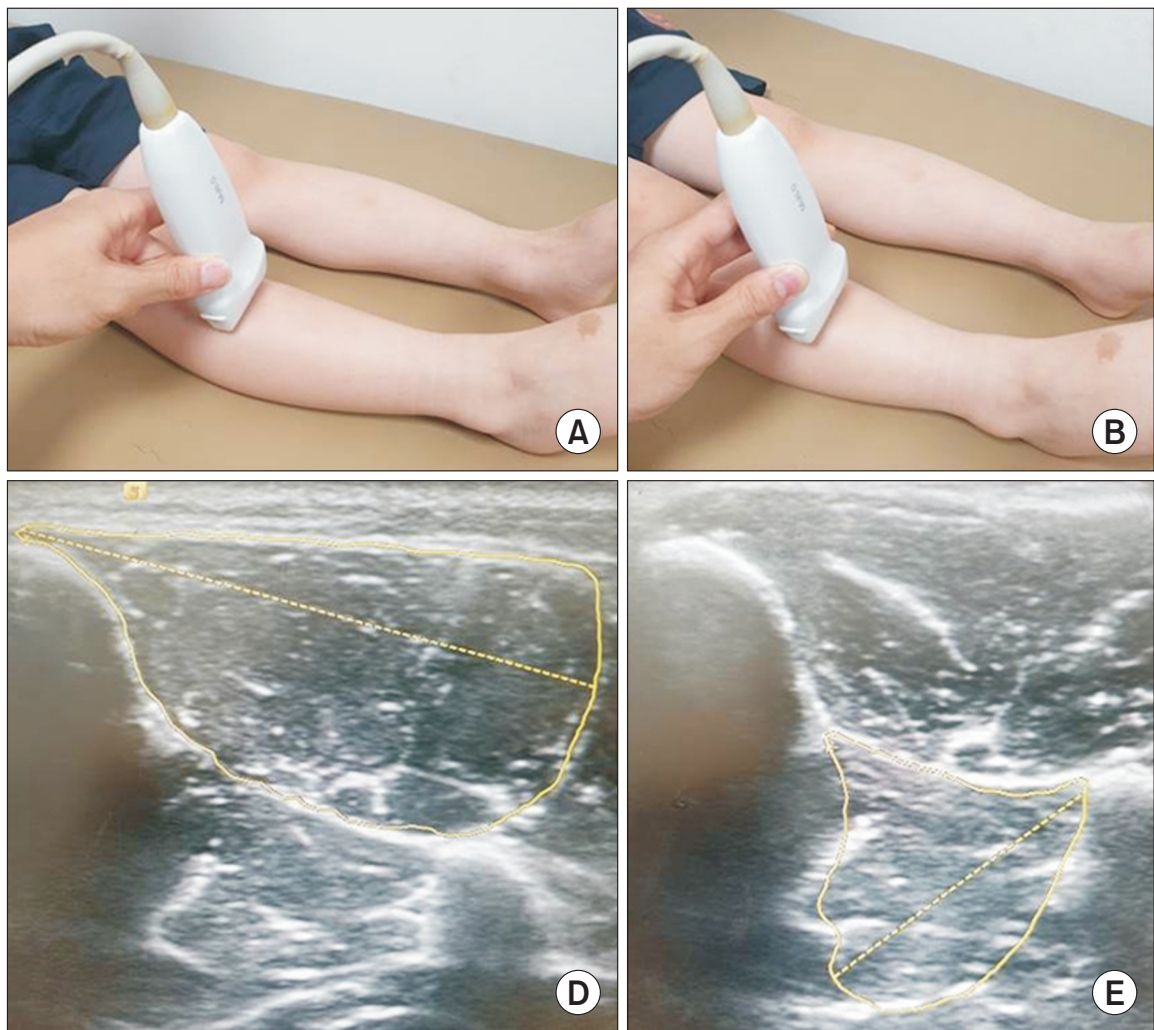



Fig. 1. Ultrasonographic measurements. (A) The site of the probe to measure the TA muscle. (B) The site of the probe to measure the TP muscle, (C) The site of the probe to measure the PL muscle. (D) Cross-sectional area of TA. (E) Cross-sectional area of TP. (F) Cross-sectional area of PL. TA, tibialis anterior; TP, tibialis posterior; PL, peroneus longus. 
for measuring CSA of TP: the posterior approach and anterior approach. Both methods can accurately measure CSA without much difference [26], and in this study, the anterior approach was used to facilitate data collection. CSA was measured three times for each muscle, and the median value was used in the analysis.

As muscles grow during childhood and adolescence and the rate of growth varies depending on age and sex [14], the ratio was calculated using the values measured with ultrasonography and the changes in the ratio of each muscle to the total area of the TA, TP, and PL were compared.

TA ratio $=$ CSA of TA $/(C S A$ of TA + CSA of TP + CSA of PL $)$

$\mathrm{TP}$ ratio $=\mathrm{CSA}$ of TP $/(\mathrm{CSA}$ of TA + CSA of TP + CSA of PL $)$

$\mathrm{PL}$ ratio $=\mathrm{CSA}$ of $\mathrm{PL} /(\mathrm{CSA}$ of TA $+\mathrm{CSA}$ of $\mathrm{TP}+\mathrm{CSA}$ of $\mathrm{PL})$

\section{Secondary outcome}

RCSP angle: The RCSP angle was used to assess foot posture. The RCSP angle is the angle that the calcaneal bisection, which is marked by connecting the dots bisecting the calcaneus using a bimanual technique while

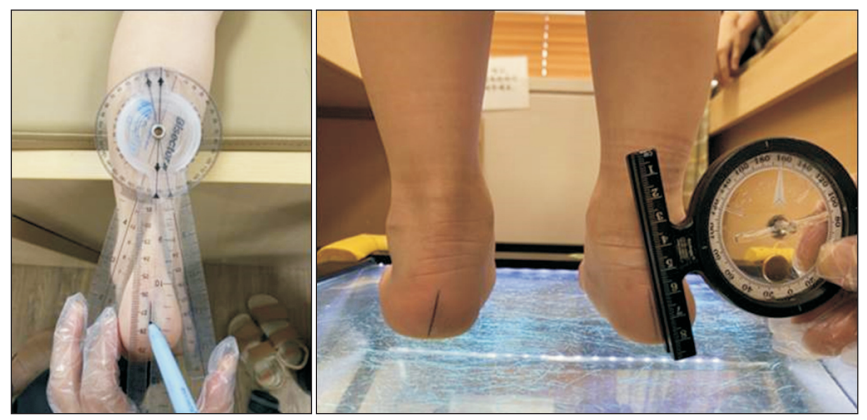

Fig. 2. Measurement of the resting calcaneal stance position angle. the patient is in the prone position, makes in relation to a vertical line to a surface while the patient is standing straight on a flat surface.

A positive RCSP angle in the standing position indicates supination, while a negative angle indicates pronation [14,25,27,28] (Fig. 2).

\section{Radiographic measurements}

Foot radiographs were acquired to assess the structural and anatomical deformities of the feet in patients with flatfoot. Radiographs were obtained in the anteriorposterior and lateral views while the patient was standing straight with shoes off. CP, MA, TNCA and TCA were measured, three times, using radiography $[14,25,27,29$ $33]$, and the median values were used in the analysis. CP was defined as the angle between the bottom line of the foot and the inferior line of the calcaneus on the lateral view of the foot radiograph. The smaller the angle, the more severe the flatfoot. MA was defined as the angle between the line bisecting the neck and head of the talus and the line bisecting the head of the talus and neck of the first metatarsal. If the apex of the angle was directed dorsally, MA was positive; if the apex was directed in the plantar direction, MA was negative. The greater the angle, the more severe the flatfoot. TNCA was defined as the angle between the line along the articular surface of the talus and the line along the articular surface of the navicular bone on the anterior-posterior view of the foot radiograph. TNCA was used to assess forefoot abduction at the midtarsal joint [34]. TCA was defined as the angle between the line along the axis of the calcaneus and the line along the axis of the talus on the anterior-posterior view of the foot radiograph. TCA was used to assess the hindfoot valgus and varus [22] (Fig. 3).
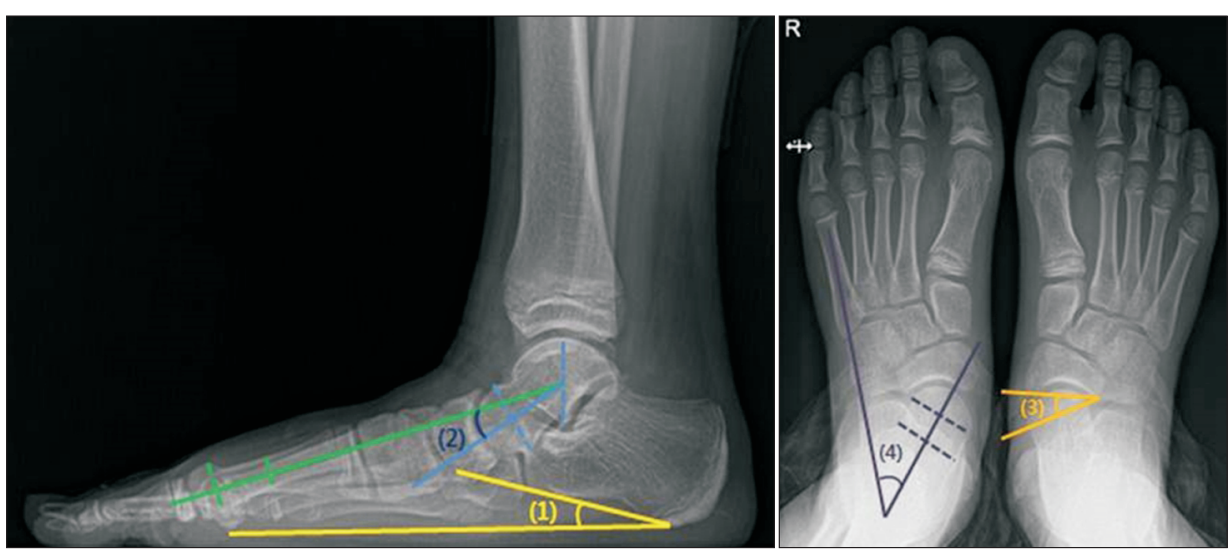

Fig. 3. Radiographic measurements. (1) Calcaneal pitch angle. (2) Meary's angle. (3) Talonavicular coverage angle. (4) Talocalcaneal angle. 


\section{FFI}

FFI, which was developed in 1991 to assess pain and functional impairment caused by foot problems, was used to assess pain and disability due to flatfoot in this study. FFI consists of three subgroups (i.e., pain, disability, and activity limitation) and has 23 items: nine for pain, nine for disability, and five for activity limitation. Each item is scored from 0 to 10 points, with lower scores indicating less severe functional impairment [35]. A revised FFI consisting of 68 items-pain and stiffness (20 items), difficulty (20 items), activity limitation (9 items), and social/emotional issues (19 items) - was published in 2006 [36]. However, as the revised version of the FFI is longer and thus time consuming and may be difficult for children to complete, the original version translated to Korean was used to ensure compliance and reliability in this study [37].

\section{Intervention}

RFOs were custom-made for each patient to treat symptomatic flexible flatfoot (Fig. 4). After 2 weeks, when the RFO production was completed, a clinician had evaluated whether the RFOs were suitable for the patient. RFOs were worn for 12 months, and the patients were instructed to wear the RFOs indoors and outdoors, for at least 6 hours per day, as much as possible. In patients with flexible flatfoot, TA and TP strengthening exercises to neutralize the valgus or intrinsic muscle and abductor hallucis strengthening exercises to prevent flattening of the anterior arch can be performed [6]. Because these exercises can affect the development of the muscles to be measured in this study, we explained to the subjects that they should only wear RFO and not exercise concurrently during the study period.

\section{Statistical analysis}

Wilcoxon signed rank test was used to compare whether the CSA of each muscle, as measured by ultrasonography, the calculated CSA ratio, RCSP angle, radiographic measurements including CP, MA, TNCA, and TNCA, and FFI were significantly different before and after RFO application.

In addition, to assess whether the degree of change in symptoms, including pain, is correlated with the degree of change in various variables, the correlation between the delta value, which means the degree of change, was analyzed. Spearman correlation analysis was used to analyze the correlation between the delta values of FFI total and subgroups and the delta values of CSA ratio, RCSP angle, radiographic measurements before and after RFO application.

To determine whether there is a significant difference in the change in the PL ratio in the delta values of the total, pain, disability, and activity limitation of the FFI score, the delta value of the FFI score was divided into two groups by 1 point and the Mann Whitney U-test was used to compare whether there was a significant difference between the two groups.

All statistical analyses were performed using IBM SPSS (version 25.0; IBM Corp., Armonk, NY, USA). The level of statistical significance was set at $\mathrm{p}<0.05$.

\section{RESULTS}

Among the patients who visited the outpatient clinic during the study period, 110 satisfied the inclusion criteria for flatfoot. Thirty-one children aged $<10$ years were excluded from the study. Of the remaining 79 patients, 76 had flexible flatfoot. Twenty-seven patients with no

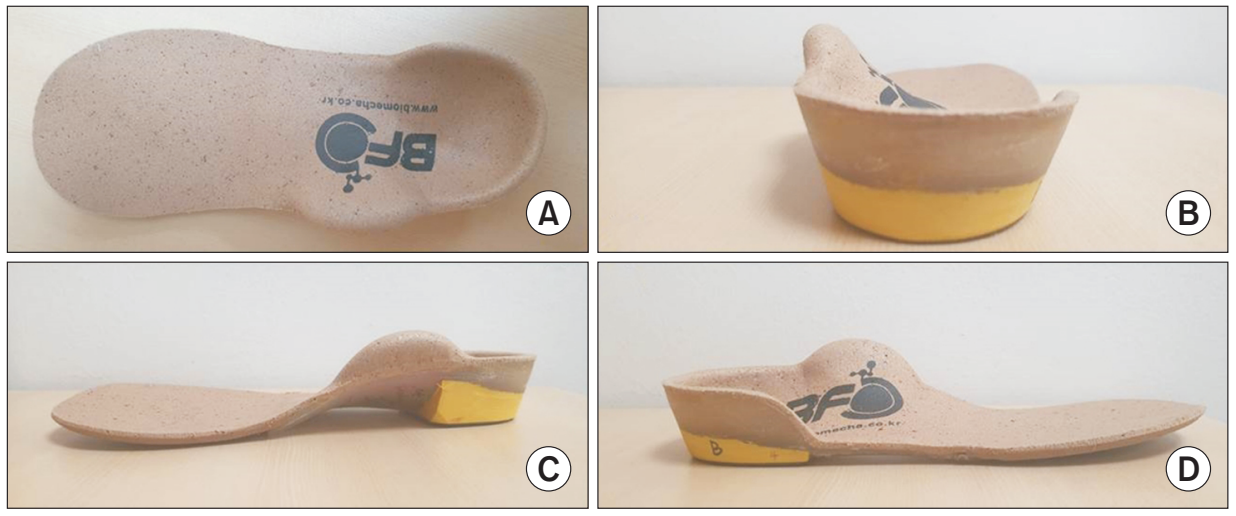

Fig. 4. Custom-made rigid foot orthosis (RFO). (A) Superior view. (B) Posterior view. (C) Medial view. (D) Lateral view of the RFO. 


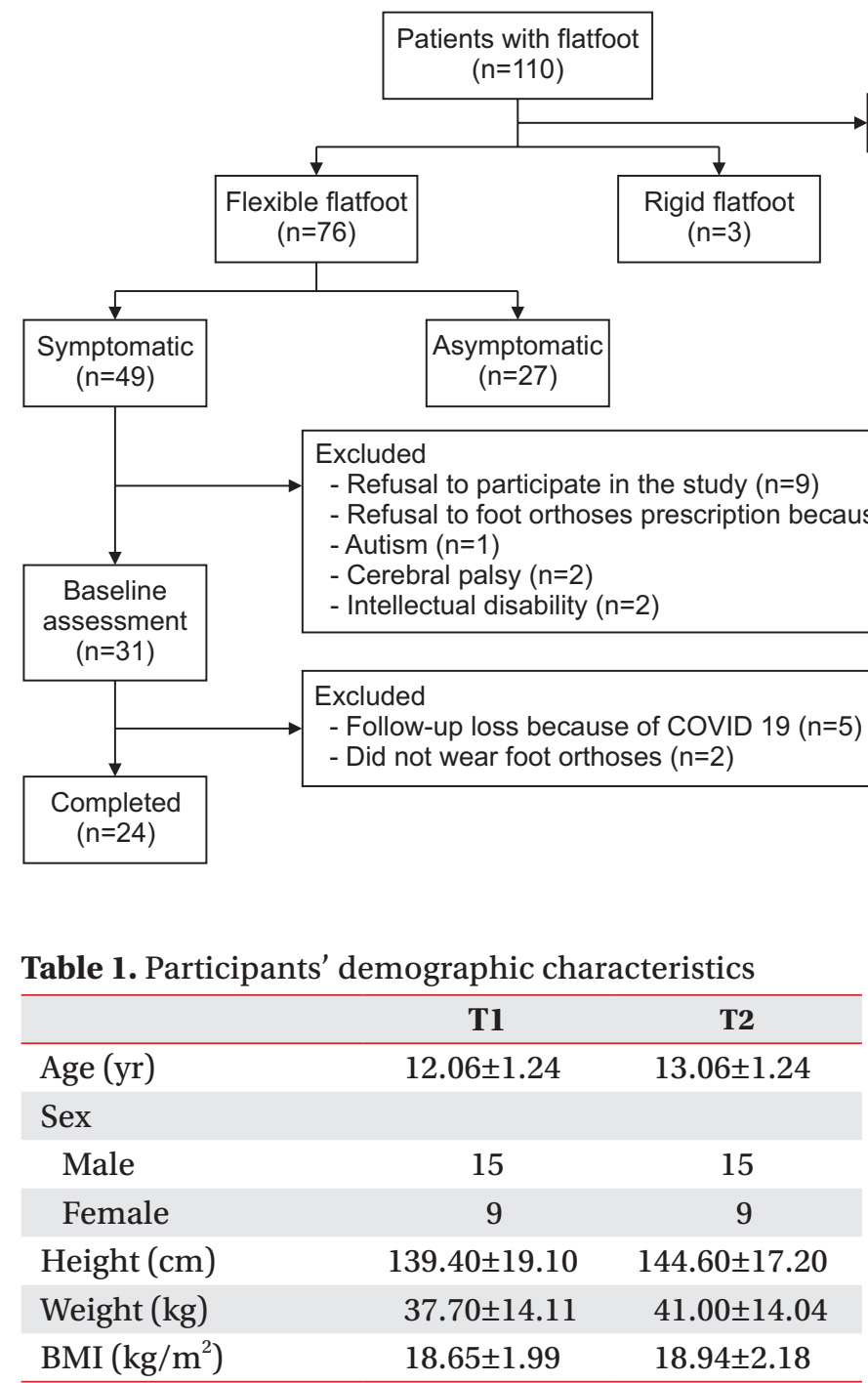

Values are presented as mean \pm standard deviation.

$\mathrm{T} 1$, before rigid foot orthosis application; T2, 12 months after rigid foot orthosis application; BMI, body mass index.

symptoms, such as pain, were also excluded. Of the remaining 49 patients with symptomatic flexible flatfoot, nine refused to participate in the study, four refused to wear foot orthoses for financial reasons, one had autism, two had cerebral palsy, and two had intellectual disability; these were excluded. The remaining 31 patients underwent the initial assessment. Five patients were lost to follow-up, and two did not wear their foot orthoses after the prescription. Twenty-four patients (15 boys and 9 girls) were included in this study (Fig. 5). Data of both feet from the 24 patients were obtained and 48 data were analyzed. Table 1 shows the demographic character-
Age $<10$ years $(n=31)$
Fig. 5. Study flow chart. COVID-19, coronavirus disease 2019.

istics of the patients. The mean age of the patients was $12.06 \pm 1.24$ years at the time of study participation and $13.06 \pm 1.24$ years at the time of reassessment at 12 months after RFO application.

Table 2 shows the CSAs of the TA, TP, and PL, which were measured using ultrasonography, and the calculated CSA ratio of each muscle. The TA, TP, and PL showed a significant increase in CSA following RFO application (all, $\mathrm{p}<0.001)$. The TA $(\mathrm{p}=0.001)$ and TP ratio $(\mathrm{p}=0.011)$ significantly decreased and the PL ratio significantly increased after RFO application ( $\mathrm{p}=0.007)$.

Table 3 shows the RCSP angle, and the radiographic measurements, obtained before and after the application of the RFOs. The RCSP angle significantly changed from $-8.13 \pm 2.64$ (before RFO application) to $-5.63 \pm 1.89$ (after RFO application) $(\mathrm{p}=0.004)$. In the radiographic measurements, a significant change in $\mathrm{CP}$ was observed following RFO application ( $\mathrm{p}=0.012$ ). MA, TNCA, and TCA decreased after RFO application, albeit by an insignificant degree.

The FFI measured before and after the application of the RFOs, which measures pain and functional disability (Table 3). The FFI total score significantly decreased from $14.43 \pm 3.93$ to $8.81 \pm 2.23(\mathrm{p}=0.001)$. In the subgroup analysis, the pain and disability scores significantly decreased from $18.75 \pm 4.56$ to $7.62 \pm 3.72$ and from $10.12 \pm 3.87$ to $8.06 \pm 2.46$, respectively $(\mathrm{p}=0.001$ and $\mathrm{p}=0.002$, respec- 
Table 2. Comparison of CSA and the ratio of each muscle to total ankle invertor and evertor muscles before and after RFO application

\begin{tabular}{lcccc}
\hline & T1 & T2 & t & p-value \\
\hline CSA of TA $\left(\mathrm{cm}^{2}\right)$ & $6.95 \pm 2.00$ & $8.20 \pm 1.61$ & 3.517 & $<0.001^{*}$ \\
CSA of TP $\left(\mathrm{cm}^{2}\right)$ & $2.62 \pm 0.98$ & $3.97 \pm 0.96$ & 3.516 & $<0.001^{*}$ \\
CSA of PL $\left(\mathrm{cm}^{2}\right)$ & $3.57 \pm 0.90$ & $5.72 \pm 1.12$ & 3.516 & $<0.001^{*}$ \\
TA ratio & $0.52 \pm 0.06$ & $0.47 \pm 0.03$ & -3.464 & $0.001^{*}$ \\
TP ratio & $0.22 \pm 0.06$ & $0.20 \pm 0.03$ & -1.500 & $0.011^{*}$ \\
PL ratio & $0.28 \pm 0.06$ & $0.32 \pm 0.04$ & 2.689 & $0.007^{*}$ \\
\hline
\end{tabular}

Values are presented as mean \pm standard deviation.

CSA, cross-sectional area; RFO, rigid foot orthosis; T1, before RFO application; T2, 12 months after RFO application; $\mathrm{TA}$, tibialis anterior; TP, tibialis posterior; PL, peroneus longus.

${ }^{*} \mathrm{p}<0.05$.

Table 3. Comparison of RCSP angle, radiographic measurements in foot X-ray and FFI before and after RFO application

\begin{tabular}{|lrrrl|}
\hline & T1 & T2 & t & p-value \\
\hline RCSP angle $\left(^{\circ}\right)$ & $-8.13 \pm 2.64$ & $-5.63 \pm 1.89$ & -4.699 & $0.004^{*}$ \\
\hline Radiographic measurements $\left(^{\circ}\right)$ & & & & \\
\hline CP & $12.00 \pm 3.27$ & $13.12 \pm 2.64$ & 2.348 & $0.012^{*}$ \\
\hline MA & $9.67 \pm 5.70$ & $9.20 \pm 4.24$ & 1.313 & 0.277 \\
\hline TNCA & $25.66 \pm 9.91$ & $24.19 \pm 7.32$ & 0.496 & 0.438 \\
\hline TCA & $36.04 \pm 7.34$ & $34.31 \pm 6.55$ & 0.993 & 0.310 \\
\hline Total score of FFI & $14.43 \pm 3.93$ & $8.81 \pm 2.23$ & -4.569 & $0.001^{*}$ \\
\hline Subgroup & & & & \\
\hline Pain & $18.75 \pm 4.56$ & $7.62 \pm 3.72$ & -4.459 & $0.001^{*}$ \\
\hline Disability & $10.12 \pm 3.87$ & $8.06 \pm 2.46$ & -3.506 & $0.002^{*}$ \\
\hline Activity limitation & $2.43 \pm 0.51$ & $2.31 \pm 0.47$ & -1.386 & 0.157 \\
\hline
\end{tabular}

Values are presented as mean \pm standard deviation.

RCSP, resting calcaneal stance position; FFI, foot function index; RFO, rigid foot orthosis; T1, before RFO application; T2, 12 months after RFO application; CP, calcaneal pitch; MA, Meary's angle; TNCA, talonavicular coverage angle; TCA, talocalcaneal angle.

${ }^{*} \mathrm{p}<0.05$.

tively). However, although activity limitation tended to decrease from $2.43 \pm 0.51$ to $2.31 \pm 0.47$, the decrease was not significant.

A correlation analysis was performed to examine the correlations between the amount of change in the FFI and the amount of change in the CSA ratios of the muscles, RCSP angle, and radiographic measurements (Table 4). The total FFI score was negatively correlated with the $\mathrm{PL}$ ratio $(\mathrm{r}=-0.398, \mathrm{p}=0.037)$ and positively correlated with the RCSP angle ( $\mathrm{r}=0.476, \mathrm{p}=0.009)$. For the FFI subgroups, pain was negatively correlated with the PL ratio ( $\mathrm{r}=-0.283$, $\mathrm{p}=0.047)$ and positively correlated with the RCSP $(\mathrm{r}=0.417$, $\mathrm{p}=0.017$ ). Disability was negatively correlated with the PL ratio $(\mathrm{r}=-0.314, \mathrm{p}=0.040)$ and positively correlated with the RCSP angle ( $\mathrm{r}=0.712, \mathrm{p}=0.001)$.

Table 5 shows the specific values with significant differences in the PL ratio in the delta values of total, pain, and disability of FFI. After RFO application compared to before application, the total FFI changed from 1 to 10 , the pain score from 4 to 16 , the disability score from 0 to 8 , and the activity limitation score from 0 to 1 . The delta values of the FFI total and subgroup scores were divided into two groups by 1 point and the delta values of the PL ratio between both groups were compared. As a result, the increase in the PL ratio was significantly greater in the group with a delta value of 3-10 than in the group with 
Table 4. Correlations between the degree of change in FFI and the degree of change in CSA ratios of ankle invertor and evertor muscles, RCSP angle and radiographic measurements before and after RFO application

\begin{tabular}{|c|c|c|c|c|c|c|c|c|c|}
\hline FFI & & $\Delta T A$ ratio & $\Delta T P$ ratio & $\Delta P L$ ratio & $\Delta \mathrm{RCSP}$ & $\Delta \mathbf{C P}$ & $\Delta \mathbf{M A}$ & $\triangle \mathrm{TNCA}$ & $\triangle T C A$ \\
\hline \multirow[t]{2}{*}{$\Delta$ Total } & $\mathrm{r}$ & -0.358 & 0.050 & -0.398 & 0.476 & 0.033 & 0.295 & -0.033 & -0.374 \\
\hline & $\mathrm{p}$-value & 0.173 & 0.853 & $0.037^{*}$ & $0.009^{*}$ & 0.902 & 0.268 & 0.153 & 0.153 \\
\hline \multirow[t]{2}{*}{$\Delta$ Pain } & $\mathrm{r}$ & 0.281 & 0.017 & -0.283 & 0.417 & 0.145 & -0.019 & 0.196 & 0.298 \\
\hline & p-value & 0.119 & 0.925 & $0.047^{*}$ & $0.017^{*}$ & 0.429 & 0.919 & 0.282 & 0.269 \\
\hline \multirow[t]{2}{*}{$\Delta$ Disability } & $\mathrm{r}$ & 0.129 & 0.110 & -0.314 & 0.712 & 0.344 & -0.284 & 0.030 & 0.223 \\
\hline & $\mathrm{p}$-value & 0.481 & 0.550 & $0.040^{*}$ & $0.001^{*}$ & 0.054 & 0.115 & 0.872 & 0.304 \\
\hline \multirow[t]{2}{*}{$\Delta$ Activity limitation } & $\mathrm{r}$ & 0.031 & 0.061 & -0.092 & 0.151 & 0.108 & 0.138 & 0.186 & 0.184 \\
\hline & $\mathrm{p}$-value & 0.868 & 0.738 & 0.616 & 0.411 & 0.558 & 0.450 & 0.310 & 0.313 \\
\hline
\end{tabular}

RFO, rigid foot orthosis; FFI, foot function index; CSA, cross-sectional area; TA, tibialis anterior; TP, tibialis posterior; PL, peroneus longus; RCSP, resting calcaneal stance position; CP, calcaneal pitch; MA, Meary's angle; TNCA, talonavicular coverage angle; TCA, talocalcaneal angle.

${ }^{*} \mathrm{p}<0.05$ using the Spearman correlation analysis.

Table 5. Comparison of the degree of change in PL ratio between both groups divided by cutoff value of difference in FFI total and subgroup score

\begin{tabular}{|c|c|c|c|c|}
\hline FFI & $\mathbf{n}$ & $\Delta P L$ ratio & $t$ & p-value \\
\hline$\Delta$ Total (1-2 vs. $3-10)$ & & & -3.070 & $0.001^{*}$ \\
\hline $1-2$ & 12 & $0.064 \pm 0.200$ & & \\
\hline $3-10$ & 36 & $0.103 \pm 0.028$ & & \\
\hline$\Delta$ Pain (4-7 vs. $8-16$ ) & & & -2.344 & $0.019 *$ \\
\hline $4-7$ & 14 & $0.069 \pm 0.022$ & & \\
\hline $8-16$ & 34 & $0.100 \pm 0.030$ & & \\
\hline$\Delta$ Disability (0-1 vs. $2-8)$ & & & -3.764 & $<0.001^{*}$ \\
\hline $0-1$ & 26 & $0.073 \pm 0.023$ & & \\
\hline $2-8$ & 22 & $0.113 \pm 0.025$ & & \\
\hline$\Delta \mathrm{AL}(0$ vs. 1$)$ & & & -1.090 & 0.276 \\
\hline 0 & 38 & $0.090 \pm 0.031$ & & \\
\hline 1 & 10 & $0.108 \pm 0.029$ & & \\
\hline
\end{tabular}

Values are presented as mean \pm standard deviation.

FFI, foot function index; PL, peroneus longus; AL, activity limitation.

${ }^{*} \mathrm{p}<0.05$.

a delta value of 1-2 of the FFI total score ( $\Delta$ Total: $1-2$ vs. $3-10 ; p=0.001$ ), the group with a delta value of 8-16 than in the group with a delta value of 4-7 of the pain score $(\triangle$ Pain: $4-7$ vs. $8-16 ; p=0.019)$ and the group with a delta value of 2-8 than in the group with a delta value of $0-1$ of the disability score ( $\Delta$ Disability: $0-1$ vs. $2-8 ; \mathrm{p}<0.001)$. However, there was no significant difference between the group with activity limitation score of 0 and the group with activity limitation score of 1 .

\section{DISCUSSION}

Although numerous studies on the diagnosis and treatment of flatfoot have been conducted, most of them used radiography and few have assessed and compared the foot muscles in patients with flatfoot using ultrasonography. This study was the first to identify how RFOs affect the CSA ratios of the ankle invertors and evertors, which are ankle-stabilizing muscles, in children with flatfoot and to investigate the relationship between changes in CSA of the muscles and pain. 
Similar to a previous study [14], this study examined changes in the CSA ratios of the TA, TP, and PL after RFO application. The CSA ratios of the TA and TP (ankle invertors) significantly decreased, whereas the CSA ratio of the PL (ankle evertor) significantly increased, following RFO application. In a study examining the activities of foot muscles during walking in patients with flatfoot using electromyography, Murley et al. [9] reported large activities in the TA and TP and small activities in the PL. Shin et al. [14] compared the CSAs of the ankle invertors and evertors between children with flatfoot and those without flatfoot using ultrasonography and found that those with flatfoot have greater TA and TP ratios and a smaller PL ratio. In a similar study, Sakamoto and Kudo [2] compared the thickness and CSAs of intrinsic foot muscles, such as the $\mathrm{AH}$, between a flatfoot group and a typical foot group using ultrasonography. The flatfoot group had a larger $\mathrm{AH}$, which is a forefoot adductor, and smaller abductor digiti minimi and oblique head of the adductor hallucis, which are forefoot abductors. They reported that in patients with flexible flatfoot, the activities of the TA and TP increase to compensate for MLA collapse, which causes foot eversion when the foot touches the ground in the stance phase of a gait cycle. Based on this report, we hypothesized that RFOs would prevent foot overpronation during the loading response in the stance phase, thereby reducing the activities of the TA and $\mathrm{TP}$, reducing the TA and TP ratios, and increasing the PL ratio. Our findings supported our hypothesis and confirmed that the TA and TP ratios decrease and the PL ratio increases after RFO application. At 12 months after RFO application, the CSAs of the TA and TP slightly increased from $6.95 \pm 2.00$ to $8.20 \pm 1.61$ and from $2.62 \pm 0.98$ to $3.97 \pm 0.96$, respectively, whereas the PL significantly increased from $3.57 \pm 0.90$ to $5.72 \pm 1.12$, which may be because, contrary to the TA and TP that had developed excessively to compensate for foot overpronation in patients with flatfoot, the PL was underdeveloped. As the foot posture was corrected by the RFOs, the TA, TP, and PL may have begun to grow normally, thereby resulting in a slight increase in the CSAs of the TA and TP and a greater increase in the CSA of the PL to catch up with normal growth.

Evidence of whether foot orthoses can effectively treat flatfoot is lacking. Some studies have reported that foot orthoses are uncomfortable and ineffective [38] and that wearing foot orthoses can even lower self-esteem in children [39]. However, Lee et al. [18], who assessed the effect of foot orthoses on foot pain and balance in 24 patients aged $\geq 6$ years with flexible flatfoot, reported a significant pain reduction 1-3 months after wearing foot orthoses. Shin et al. [14] investigated the correlations among the severity of symptoms including pain, the ratio of each muscle, and radiographic measurements. They found that the FFI is significantly correlated with the TA ratio, $\mathrm{PL}$ ratio, $\mathrm{CP}, \mathrm{MA}$, and TNCA and that the FFI is most strongly correlated with the TNCA and PL ratio, thereby suggesting that the TNCA and PL ratio may be used to predict symptom severity. Based on these findings, they suggested that orthoses that limit forefoot abduction or exercise therapy that strengthens the PL may reduce symptoms, such as pain, in patients with flexible flatfoot. In our study, we investigated whether RFOs can reduce pain in patients with flexible flatfoot and studied the correlations between the FFI, CSA ratios of muscles, and radiographic measurements before and after RFO application. We found that RFO enhances foot function impairment due to flatfoot as evidenced by FFI reduction after RFO application. Specifically, pain and disability were reduced. Based on the correlation analysis, we found that the greater the change in the PL ratio and the RCSP angle, the lesser the pain and disability. In detail, the increase in PL ratio was statistically significantly higher for those with FFI total scores reduced by more than 3 points, FFI pain scores reduced by more than 8 points or FFI disability scores reduced by more than 2 points. However, in this study, the sample size was small, so the difference in the number of people between the two groups divided by a specific delta value was large. Further studies with a large number of patients are needed to determine the definitive cutoff value of difference. Ankle-stabilizing muscles develop disproportionately in patients with flexible flatfoot, which in turn causes pain and discomfort when walking indoors or outdoors or going up and down stairs. We believe that RFOs could induce normal muscle growth, thereby reducing pain and, subsequently, disability.

In this study, we assessed the RCSP angle and radiographic measurements, including $\mathrm{CP}, \mathrm{MA}$, TNCA, and TCA, as secondary outcomes. Lee et al. [40] examined the changes in the RCSP angle induced by an insole in 66 patients with flexible flatfoot and observed a significant improvement in the RCSP angle 24 months after RFO appli- 
cation. However, they did not examine changes in the CP. Bok et al. [22] studied the effect of custom-made RFOs in 39 patients with flatfoot aged $\geq 6$ years and reported a significant increase in the CP and RCSP angle 12-18 months after RFO application. Ahn et al. [27] compared the effect between RFOs and talus control foot orthoses in 40 children with flexible flatfoot and reported significant improvements in the RCSP angle and CP 12 months after RFO application. Similarly, in our study, significant improvements in the CP and RCSP angle were observed 12 months after RFO application. In this study, it was found that it was statistically significant that the $\mathrm{CP}$ changed from mean $12^{\circ}$ before RFO application to mean $13.12^{\circ}$ after RFO application. However, a change of $1.12^{\circ}$ is less clinically significant and may be a value that can vary as a result of measurement error. Further studies with more patients are needed to confirm clinically meaningful results.

This study has several limitations. First, the sample size was relatively small. It was difficult to enroll patients in this study as the study targeted school-age children, who require parents' consent to participate in the study, and because of concerns among the patients and parents about frequent visits to the hospital during the coronavirus disease 2019 (COVID-19) pandemic. Additionally, numerous patients were lost to follow-up after the initial assessment. Second, this study did not include a control group. There is limited information on how the CSA ratio of the ankle invertor and evertor naturally changes in the general population or children without flexible flatfoot. While clearer results may have been obtained regarding the effect of RFOs by comparing patients with RFO (treatment group) and those without (control group) among patients experiencing pain due to flatfoot, monitoring patients who are experiencing pain without management such as RFO application was clinically impossible. Third, the measurement locations may vary as bones, such as the tibia and fibula, grew over time, thereby introducing measurement biases. Fourth, we did not investigate the number of hours per day that each patient wore the RFOs. Most patients use RFOs when they are outdoors; thus, we expect that the actual application time would be shorter because outdoor activities were limited during the study period by environmental factors, such as the COVID-19 pandemic. Finally, muscles are three-dimensional structures; the CSA of the muscles measured using ultraso- nography in this study was two-dimensional. In addition, the measurements may vary even if the ultrasonographic examination is performed at the same location because the shape and volume of the muscles differ for each person. Hence, further research with three-dimensional reconstruction using computed tomography or magnetic resonance imaging is needed to precisely measure and compare muscle changes.

In conclusion, this study was the first to compare the CSAs of the ankle invertors and evertors before and after RFO application in patients with flatfoot. Our study confirmed that RFOs might reduce the compensatory activities of the ankle invertors, thereby increasing the PL ratio, and that pain decreases as the PL ratio increases. Therefore, RFOs are effective in reducing pain by inhibiting abnormal development caused by overuse of the ankle invertor and facilitating normal development of the ankle-stabilizing muscles among patients with flatfoot. However, whether the ratios of TA, TP, and PL after applying foot orthoses in patients with flatfoot are similar to those in people with normal feet warrants further investigation.

\section{CONFLICT OF INTEREST}

No potential conflict of interest relevant to this article was reported.

\section{AUTHOR CONTRIBUTION}

Conceptualization: Cho DJ, Ahn SY, Bok SK. Methodology: Cho DJ, Bok SK. Formal analysis: Cho DJ, Ahn SY, Bok SK. Investigation: Cho DJ, Bok SK. Resources: Bok SK. Data curation: Cho DJ, Ahn SY, Bok SK. Writingoriginal draft: Cho DJ. Writing-review and editing: Cho DJ, Ahn SY, Bok SK. Visualization: Cho DJ, Ahn SY. Supervision: Bok SK. Project administration: Bok SK. All authors have read and agreed to the published version of the manuscript.

\section{REFERENCES}

1. Aenumulapalli A, Kulkarni MM, Gandotra AR. Prevalence of flexible flat foot in adults: a cross-sectional study. J Clin Diagn Res 2017;11:AC17-20.

2. Sakamoto K, Kudo S. Morphological characteristics 
of intrinsic foot muscles among flat foot and normal foot using ultrasonography. Acta Bioeng Biomech 2020;22:161-6.

3. Sadeghi-Demneh E, Melvin JM, Mickle K. Prevalence of pathological flatfoot in school-age children. Foot (Edinb) 2018;37:38-44.

4. Pfeiffer M, Kotz R, Ledl T, Hauser G, Sluga M. Prevalence of flat foot in preschool-aged children. Pediatrics 2006;118:634-9.

5. Jandric SD, Kragulj P. Scoliosis, life style and low back pain in adolescents. Vojnosanit Pregl 2021;78:987-93.

6. Halabchi F, Mazaheri R, Mirshahi M, Abbasian L. Pediatric flexible flatfoot; clinical aspects and algorithmic approach. Iran J Pediatr 2013;23:247-60.

7. Amoozadeh F, Kazemian G, Rasi AM, Kazemi P, Safaeinik F, Khazanchin A. Surveying the relationship between flatfoot and chronic mechanical low back pain. Indian J Fundam Appl Life Sci 2015;5:79-83.

8. Mosca VS. Flexible flatfoot in children and adolescents. J Child Orthop 2010;4:107-21.

9. Murley GS, Menz HB, Landorf KB. Foot posture influences the electromyographic activity of selected lower limb muscles during gait. J Foot Ankle Res 2009;2:35.

10. Han K, Bae K, Levine N, Yang J, Lee JS. Biomechanical effect of foot orthoses on rearfoot motions and joint moment parameters in patients with flexible flatfoot. Med Sci Monit 2019;25:5920-8.

11. Murley GS, Tan JM, Edwards RM, De Luca J, Munteanu SE, Cook JL. Foot posture is associated with morphometry of the peroneus longus muscle, tibialis anterior tendon, and Achilles tendon. Scand J Med Sci Sports 2014;24:535-41.

12. Shin JB, Kim SW, You S, Lee SK, Kim HS. Biomechanic analysis of lower extremities in children and teenagers with pes planus. J Korean Acad Rehabil Med 2008;32:154-9.

13. Angin S, Crofts G, Mickle KJ, Nester CJ. Ultrasound evaluation of foot muscles and plantar fascia in pes planus. Gait Posture 2014;40:48-52.

14. Shin Y, Ahn SY, Bok SK. Relationships between relative ankle muscle ratios, severity of symptoms, and radiologic parameters in adolescent patients with symptomatic flexible flat feet. Ann Rehabil Med 2021;45:123-30.

15. Evans AM, Rome K. A Cochrane review of the evidence for non-surgical interventions for flexible pedi- atric flat feet. Eur J Phys Rehabil Med 2011;47:69-89.

16. Banwell HA, Paris ME, Mackintosh S, Williams CM. Paediatric flexible flat foot: how are we measuring it and are we getting it right?: a systematic review. J Foot Ankle Res 2018;11:21.

17. Lee HJ, Lim KB, Yoo J, Yoon SW, Jeong TH. Effect of foot orthoses on children with lower extremity growing pains. Ann Rehabil Med 2015;39:285-93.

18. Lee HJ, Lim KB, Yoo J, Yoon SW, Yun HJ, Jeong TH. Effect of custom-molded foot orthoses on foot pain and balance in children with symptomatic flexible flat feet. Ann Rehabil Med 2015;39:905-13.

19. Ueki Y, Sakuma E, Wada I. Pathology and management of flexible flat foot in children. J Orthop Sci 2019;24:9-13.

20. Dars S, Uden H, Banwell HA, Kumar S. The effectiveness of non-surgical intervention (Foot Orthoses) for paediatric flexible pes planus: a systematic review: Update. PLoS One 2018;13:e0193060.

21. Unver B, Erdem EU, Akbas E. Effects of short-foot exercises on foot posture, pain, disability, and plantar pressure in pes planus. J Sport Rehabil 2019;29:43640.

22. Bok SK, Kim BO, Lim JH, Ahn SY. Effects of custommade rigid foot orthosis on pes planus in children over 6 years old. Ann Rehabil Med 2014;38:369-75.

23. Jay RM, Schoenhaus HD, Seymour C, Gamble S. The Dynamic Stabilizing Innersole System (DSIS): the management of hyperpronation in children. J Foot Ankle Surg 1995;34:124-31.

24. Whitford D, Esterman A. A randomized controlled trial of two types of in-shoe orthoses in children with flexible excess pronation of the feet. Foot Ankle Int 2007;28:715-23.

25. Youn KJ, Ahn SY, Kim BO, Park IS, Bok SK. Longterm effect of rigid foot orthosis in children older than six years with flexible flat foot. Ann Rehabil Med 2019;43:224-9.

26. Johnson AW, Bruening DA, Violette VA, Perkins KV, Thompson CL, Ridge ST. Ultrasound imaging is reliable for tibialis posterior size measurements. J Ultrasound Med 2020;39:2305-12.

27. Ahn SY, Bok SK, Kim BO, Park IS. The effects of talus control foot orthoses in children with flexible flatfoot. J Am Podiatr Med Assoc 2017;107:46-53.

28. Sobel E, Levitz SJ, Caselli MA, Tran M, Lepore F, Lilja 
E, et al. Reevaluation of the relaxed calcaneal stance position: reliability and normal values in children and adults. J Am Podiatr Med Assoc 1999;89:258-64.

29. Schon LC, Weinfeld SB, Horton GA, Resch S. Radiographic and clinical classification of acquired midtarsus deformities. Foot Ankle Int 1998;19:394-404.

30. Arunakul M, Amendola A, Gao Y, Goetz JE, Femino JE, Phisitkul P. Tripod Index: diagnostic accuracy in symptomatic flatfoot and cavovarus foot: part 2. Iowa Orthop J 2013;33:47-53.

31. Bourdet C, Seringe R, Adamsbaum C, Glorion C, Wicart P. Flatfoot in children and adolescents: analysis of imaging findings and therapeutic implications. Orthop Traumatol Surg Res 2013;99:80-7.

32. Coughlin MJ, Kaz A. Correlation of Harris mats, physical exam, pictures, and radiographic measurements in adult flatfoot deformity. Foot Ankle Int 2009;30:60412.

33. Sensiba PR, Coffey MJ, Williams NE, Mariscalco M, Laughlin RT. Inter- and intraobserver reliability in the radiographic evaluation of adult flatfoot deformity. Foot Ankle Int 2010;31:141-5.

34. Moraleda L, Mubarak SJ. Flexible flatfoot: differences in the relative alignment of each segment of the foot between symptomatic and asymptomatic patients. J Pediatr Orthop 2011;31:421-8.

35. Budiman-Mak E, Conrad KJ, Roach KE. The Foot Function Index: a measure of foot pain and disability. J Clin Epidemiol 1991;44:561-70.

36. Budiman-Mak E, Conrad K, Stuck R, Matters M. Theoretical model and Rasch analysis to develop a revised Foot Function Index. Foot Ankle Int 2006;27:519-27.

37. In TS, Jung JH, Kim K, Jung KS, Cho HY. The reliability and validity of the Korean version of the foot function index for patients with foot complaints. J Phys Ther Sci 2017;29:53-56.

38. Staheli LT. Planovalgus foot deformity: current status. J Am Podiatr Med Assoc 1999;89:94-9.

39. Driano AN, Staheli L, Staheli LT. Psychosocial development and corrective shoewear use in childhood. J Pediatr Orthop 1998;18:346-9.

40. Lee EC, Kim MO, Kim HS, Hong SE. Changes in resting calcaneal stance position angle following insole fitting in children with flexible flatfoot. Ann Rehabil Med 2017;41:257-65. 